\title{
EOSTAR Pro: a flexible extensive library to assess EO sensor performance
}

\author{
Alexander M.J. van Eijk*a ${ }^{\mathrm{a}}$, Marianne A.C. Degache ${ }^{\mathrm{a}}$, Dirk-Jan J. de Lange ${ }^{\mathrm{a}}$, Steve M. Hammel ${ }^{\mathrm{b}}$ and \\ Dimitris S. Tsintikidis ${ }^{\mathrm{b}}$ \\ ${ }^{a}$ TNO Defense, Security and Safety, P.O.Box 96864, 2509 JG The Hague, The Netherlands; \\ bPAWAR Systems Center Pacific, code 5548, San Diego CA, USA
}

\begin{abstract}
Efficient military operations require insight in the capabilities of the available sensor package to reliably assess the operational theatre, as well as insight in the adversary's capabilities to do the same. This paper presents the EOSTAR model suite, an end-to-end approach to assess the performance of electro-optical sensor systems in an operational setting. EOSTAR provides the user with coverage diagrams ("where can I see the threat?") and synthetic sensor images ("how do I perceive the threat?"), and allows assessing similar parameters for threat sensors. The paper discusses the elements of EOSTAR and outlines a few of the possible applications of the model.
\end{abstract}

Keywords: Electro-optics, sensor performance, TDA, propagation

\section{INTRODUCTION}

The military operational theatre has changed significantly over the last decades. In the post cold war era, international missions, law enforcement, humanitarian aid and peace keeping operations require flexible military organizations with the ability to operate efficiently in any geographical region and in any environment. Efficient operations require the capability to achieve reliable operational situational awareness, which in turn relies heavily on the most optimal deployment of sensor systems.

The performance of sensor systems is often not determined by technical limitations, but by the environment in which they are operated. In some cases, the impact of the environment is evident: rain, snow and fog limit the effective range of electro-optical sensors. In other cases, the relation with the environment is more subtle: temperature and humidity gradients in the atmosphere may cause ducting conditions allowing radars sometimes to see beyond the horizon, and sometimes to have a shorter range. To further complicate matters, the environment impacts differently on various sensors and sensor bands. Conditions that severely limit the performance of radars may be beneficial for electro-optical systems and vice versa.

It is crucial to assess the performance of sensors in a particular environment, as only this provides an answer to the question up to what range the sensors would be able to pick up a threat. Vice versa, it also answers the question up to what range the own platform is visible to threat sensors. The adverse impact of the environment on sensor performance requires an answer to the question "if I do not see the threat, does that mean that the threat is not there, or are my sensors not able to pick up the threat?" Such answers can be provided by tools assessing sensor performance as function of environmental conditions.

This paper discusses one of these tools: the model suite EOSTAR, an acronym for Electro-Optical System Transmission and Ranging. The tool focuses on electro-optical (infrared) sensor systems deployed in the maritime near-surface environment, although the most recent advances allow for higher-altitude scenarios and (limited) land-based scenarios.

*lex.vaenijk@tno.nl, phone: +31 70374 0451, fax: +31 70374 0654,www.tno.nl

Optics in Atmospheric Propagation and Adaptive Systems XIII, edited by Karin Stein, John D. Gonglewski, Proc. of SPIE Vol. 7828, 782804 · @ 2010 SPIE · CCC code: 0277-786X/10/\$18 · doi: 10.1117/12.868184 


\section{EOSTAR MODEL OVERVIEW}

\subsection{End-to-end approach}

Answering the question "can I see the threat?" requires more than a technical evaluation of the sensor's capabilities. The sensor is not an isolated asset, but it is deployed in a scenario (see Figure 1 on next page). The sensor views a target against a background, which may induce clutter that in turn leads to a reduction in contrast. The target may try to reduce its signature by camouflage and/or countermeasures, and the atmosphere between the target and the sensor may further degrade the signal intensity and contrast. Once registered by the sensor, the signal may be conditioned by image enhancement techniques. Finally, an operator or automated detection algorithm must make the decision if the target is detected or identifiable.

In the context of the multitude of processes outlined above, EOSTAR goes beyond the definition of "sensor performance" in a strictly technical sense. EOSTAR evaluates the sensor's performance in a relevant military operational context, i.e., against a specific threat in a specific environment, and by considering the complete observation chain. Therefore, we prefer characterizing EOSTAR as an end-to-end approach.

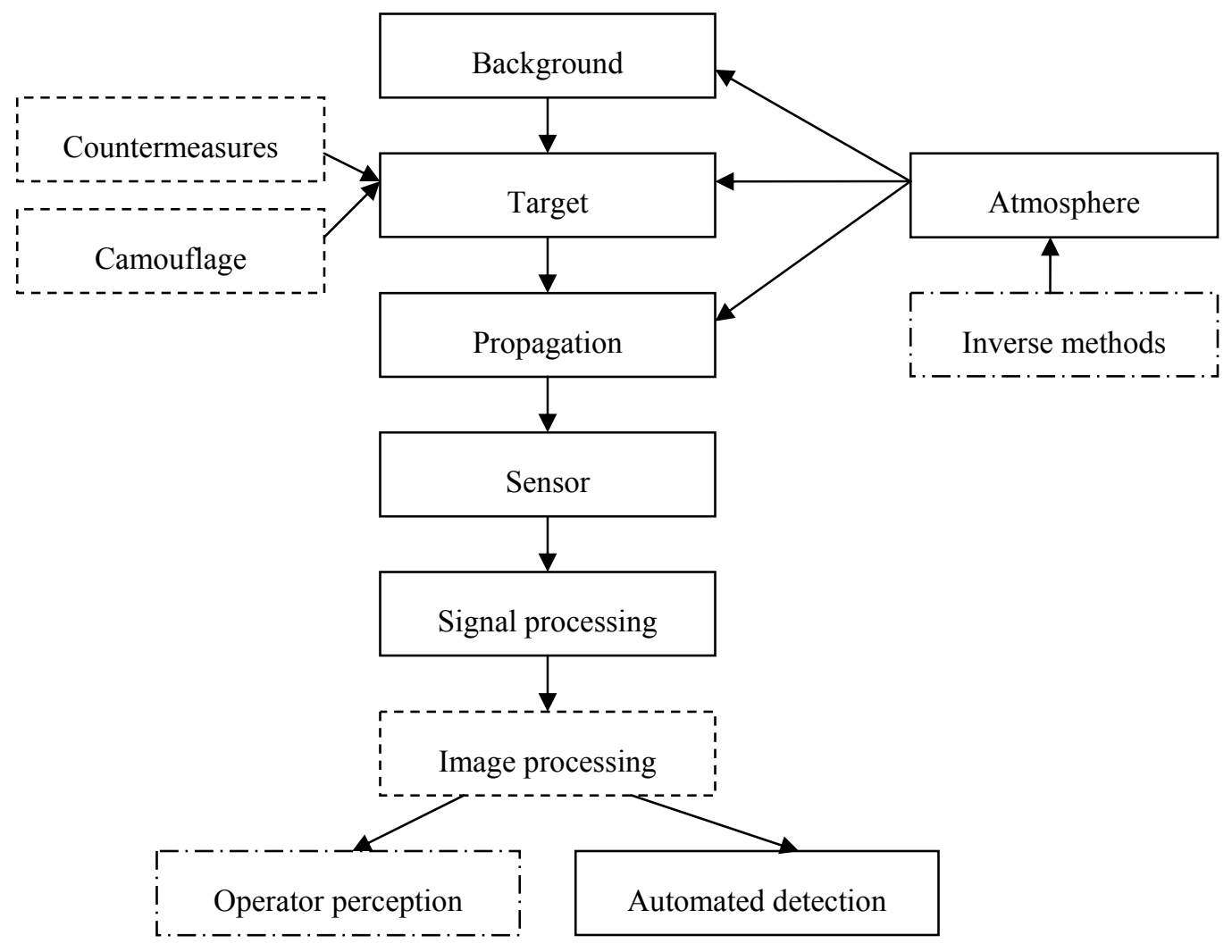

Figure 1: End-to-end approach of EOSTAR Pro. Dashed boxes represent units that have not yet been integrated in the package. The dashed-dotted box represents a unit that is in the process of being integrated. 


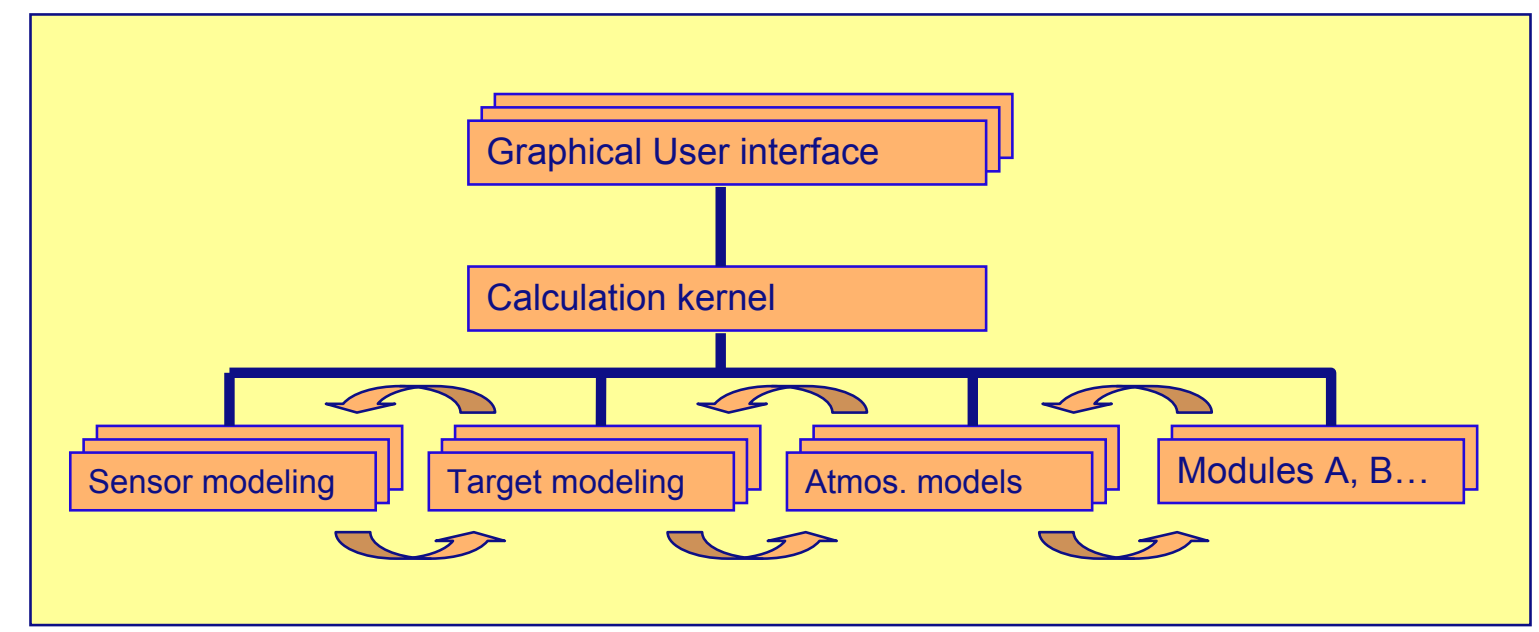

Figure 2: Modular structure of EOSTAR Pro. The central controller (kernel) assures the communication between the low-level models and the graphical user interface.

\subsection{Modular structure}

The EOSTAR model suite is fully modular. The central element of the code is the calculation kernel (see Figure 2), which assures the communication between the low-level models and a graphical user interface (GUI). The kernel evaluates the information request from the user and invokes the proper models in the proper order to provide the answer that is returned to the GUI.

The central role of the kernel offers several unique features. First of all, the kernel owns all data of the low-level models. When the GUI passes a request, the kernel may thus identify the data that are up-to-date and those that need to be (re)calculated. This enables an efficient run-mode in which the kernel handles changes in a single input parameter (e.g., the distance of a target) with a minimum of additional calculations.

The central role of the kernel also allows for efficient management of the low-level models, e.g., to update an existing model or to add a new model. All communication between the kernel and the low-level models passes through interfaces, which implies that the models remain true stand-alone codes (and thus can be provided as DLLs). On the other hand, the interfaces permit each model access to all of the data from the other models, if necessary.

Finally, the kernel decouples the graphical user interface from the actual calculations. The GUI only serves to collect the user's input requests and to present EOSTAR's output to the user. Communication between the kernel and the GUI also takes place through interfaces only. These interfaces provide access to any parameter that is present in the datapools of the kernel, but the GUI is not required to set (input) or to display (output) all of these parameters. This allows creating a series of GUIs tailored to specific applications (e.g., surveillance or soft-kill efficiency, see chapter 4), and thus, with tailored input (target selection, level of detail in specifications) and output (coverage diagrams, tables, graphs, synthetic images, etc).

The GUI is not a mandatory element of EOSTAR. It is equally possible to use the kernel and the underlying low-level models as a DLL. Input is provided through data files, and likewise, output is returned in data files or data structures in memory. This allows integrating EOSTAR in third-part software packages ("EOSTAR inside"), in which EOSTAR provides a specific intermediate product (e.g., the synthetic sensor image for a missile seeker).

\subsection{EOSTAR building blocks}

EOSTAR contains models that have been individually described and validated. Sea and sky backgrounds near the horizon are generated by the TNO Marine Infrared Background Simulator (MIBS). ${ }^{1}$ MIBS yields spatially and temporally resolved background radiant intensities and can handle clouds, sun glint and coastal backgrounds. An extension of the background module to accommodate near-infrared and visible wavelength bands is currently considered. 
The target module is centered around the TNO Electro-Optical Signature Model (EOSM). ${ }^{2}$ EOSM solves the full heatbalance equation for a three-dimensional facetted target, taking into account the sky radiance, the incident solar irradiation and the target's geometrical, structural properties as well as its steady movements. A separate module allows adding a stack plume to the target. EOSM thus generates the spectrally and spatially resolved radiant intensity of the target.

The propagation module handles transmission losses, refraction effects and turbulence. These effects cause contrast loss and image degradation over the path between target and sensor. The module includes the well-known MODTRAN code $^{3}$ the only licensed third-party code required to run EOSTAR. It is primarily used to calculate spectrally resolved molecular transmission. The propagation module further includes the Advanced Navy Aerosol Model (ANAM), ${ }^{4}$ a Snell's Law Ray Tracing Scheme (SLARTS), ${ }^{5}$ a module to calculate path radiance based on Kirchhoff's' law, and a module to evaluate turbulence effects (beam wander and scintillation). ${ }^{6}$

The sensor and signal processor building blocks are contained in the Integrated Scene Image Simulator (ISIS). ISIS handles both the optical part of the sensor (incident radiance on detector) and the signal processing. The automated detection module currently consists of an image-based algorithm. This algorithm compares radiant intensities of target and background and supplies line-integrated average signal-to-noise ratios. The operator perception model is EOVISTA, ${ }^{7}$ which uses the Triangle Orientation Discrimination (TOD) method, an improvement on the well-known Johnson criterion.

The environment that determines background properties, target signatures and propagation effects is generated in the atmosphere building block. EOSTAR currently provides a vertically inhomogeneous atmosphere. To this end, EOSTAR provides two micro-meteorological models (Turbulence And Refraction Model Over the Sea, TARMOS, ${ }^{6}$ and the Micro-Meteorological Preprocessor, $\mathrm{MPP}^{8}$ ) to generate vertical profiles of primary atmospheric variables such as wind speed and temperature on the basis of surface-based observations. In addition, external meteorological profiles can be read from radiosondes or mesoscale meteorological models. The latter input data source allows for the introduction of horizontally inhomogeneous atmospheres in EOSTAR, which is currently underway.

Two additional building blocks, for the application of inverse methods and for customizing target geometries and properties, will be discussed in chapter 3 .

\subsection{Major output products}

As mentioned above, the kernel offers access to each and every parameter and variable of EOSTAR, allowing for a wide range of output products. Based on the feedback of initial EOSTAR users, two major output products were defined: coverage diagrams and synthetic sensor images (see figure 3, next page).

The synthetic sensor image (bottom-right in figure 3) provides the operator with an impression of how a particular target at a given distance in a specific atmosphere will be viewed by the sensor. The image includes the background and all propagation effects, and thus answers the question "how does the target appear on my sensor?" The modular structure of EOSTAR also allows turning on or off individual propagation effects such as turbulence or transmission losses. In this manner, a scientist may infer the impact of such effects on image quality.

The coverage diagrams (bottom-left of figure) provide the operator with spatial information about the sensor's performance. Coverage diagrams of detection and/or classification distance in green-yellow-red inform the operator at a glance about the effective range at which the sensor can perform these tasks against a specific threat. EOSTAR provides coverage diagrams in range-azimuth and in range-height and thus answers the question "where can I see the threat?". For more scientific applications, EOSTAR can also provide coverage diagrams of underlying parameters, such as aerosol transmission, path radiance, target contrast, etc.

\subsection{Graphical user interface}

Figure 3 (next page) shows the standard GUI of EOSTAR 1.3.0. This GUI was developed in close cooperation with the (military) users of the model. The top part handles the input specification: sensor, target, and environment. All three elements can be pre-specified in three databases, thereby offering the user the possibility to fully specify EOSTAR's input by combining sensor, target and environment from pull-down menus. Alternatively, the user can customize a specific scenario (sensor, target, environment), e.g., by changing the focal length of a pre-defined sensor. As discussed above, the user may read environmental parameters from radio soundings or mesoscale meteorological models. Although the GUI proves very intuitive and easy to work with, a specific "expert" user mode was added. While a regular 


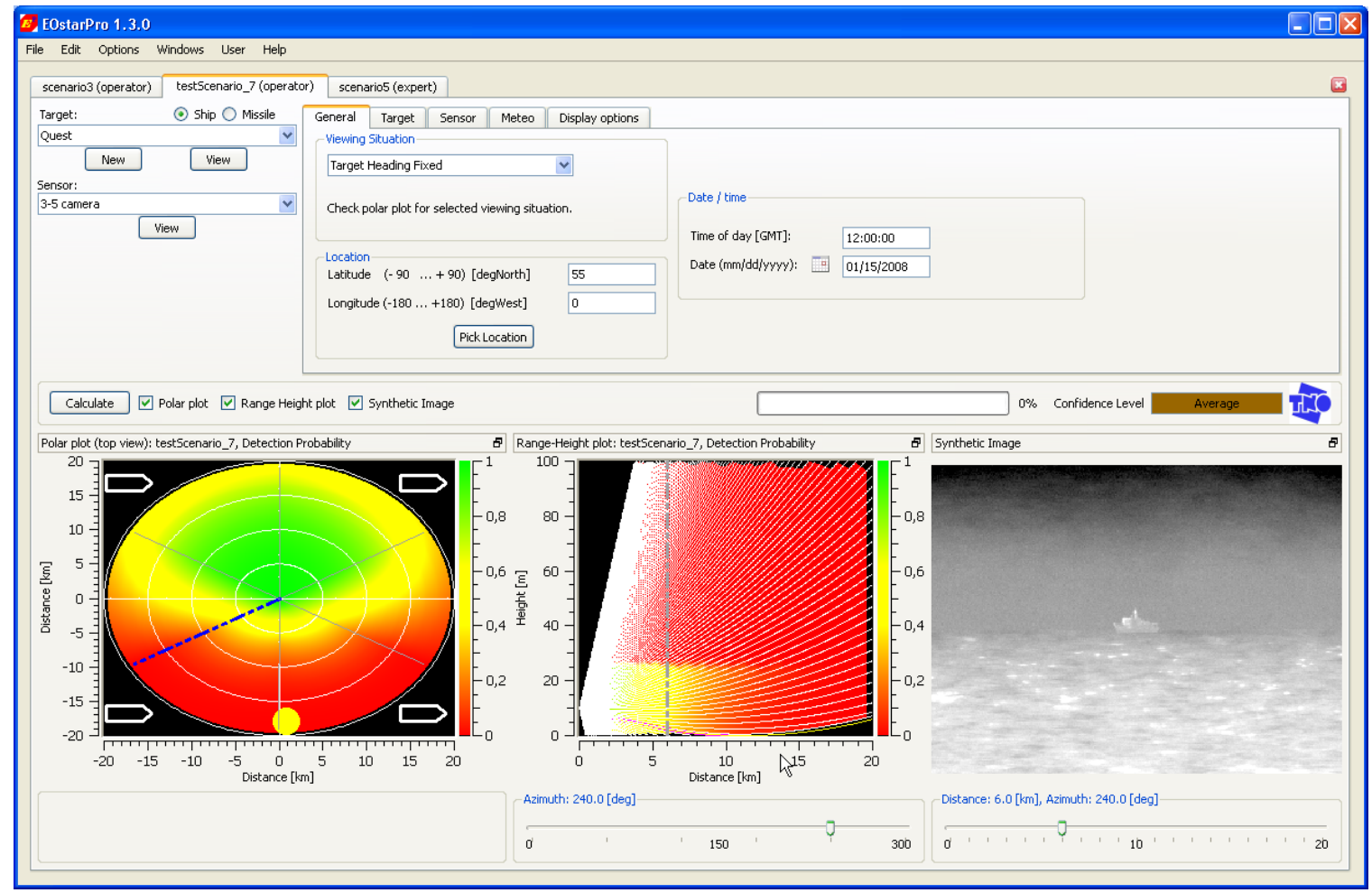

Figure 3: EOSTAR Pro 1.3.0 GUI showing the input modules (top) and major output products (bottom). From left to right: range-azimuth coverage diagram, range-height diagram, and synthetic sensor image

operator can access the databases, customize the scenario, and run the model, the expert has additional rights allowing managing of databases and selection of scientific options to tailor individual low-level models.

The output products of the standard GUI have already been discussed in the previous section. Here, it is useful to point out the intuitive arrangement of output products. The range-azimuth coverage diagram on the left provides a spatial view of the sensor's performance around the platform on which the sensor is mounted. The range-height coverage diagram in the middle resolves the vertical profile of sensor performance along a radial selected by the user and indicated by the dashed blue line (e.g., at $240^{\circ} \mathrm{N}$ in Figure 3) in the range-azimuth diagram. Finally, the synthetic image presents a view of a target located along the radial selected in the range-azimuth diagram and at a distance indicated by the (usercontrolled) dashed gray line in the range-height diagram (e.g., at $6 \mathrm{~km}$ in Figure 3).

The GUI further contains a number of markers aiding the operator in interpreting the results. The range-azimuth diagram shows the heading of the target (symbol in the corners) and the position of the sun (yellow dot at the bottom). On the right, the middle panel of the GUI includes a performance indicator signalling the reliability of the last calculation. Obviously, the GUI provides all facilities for scaling of plots, color selections, extraction of plots, and side-by-side comparison of results of different calculations.

\section{ADDITIONAL MODULES}

\subsection{TargetBuilder}

The TargetBuilder ${ }^{9}$ is an add-on module for EOSTAR providing a graphical tool (see Figure 4, next page) to define the 3D-geometry of objects as well as their physical properties, such as internal temperature and reflection coefficients. The user may use a set of simple building blocks ("hull", "stack") to create an object from scratch, or read a more complex geometrical structure (wireframe) from file. The dimensions of these external structures can subsequently be customized, allowing to study the impact of ship design changes on signatures. Although primarily used for building ships, TargetBuilder also includes elements for designing periscopes and airplanes. 


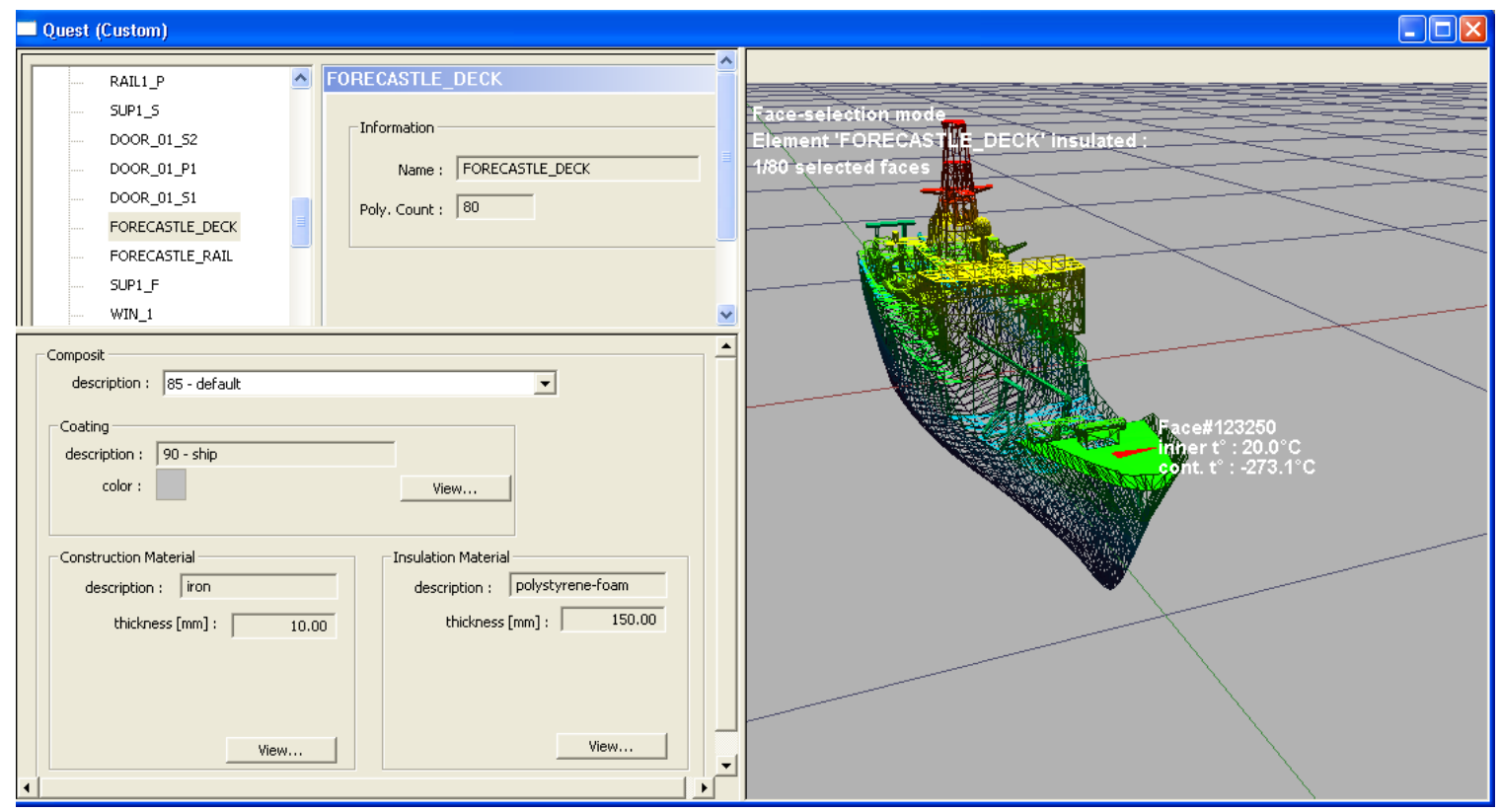

Figure 4: Add-on module TargetBuilder

Once the user has created or imported a facetted geometrical structure, the next step consists of assigning physical properties to the individual elements (or groups of elements). TargetBuilder offers a user-trainable database of composites, consisting of a construction material (e.g., "steel"), insulation material and coating.

TargetBuilder is fully interfaced with EOSTAR. Thus, the objects created by TargetBuilder can be passed on to the signature code EOSM and thereby included in the end-to-end model approach. Recent developments in TargetBuilder address the interaction between target and background, i.e., the creation of a wake by a ship or other small observable. It is not a priori clear whether the wake should be considered as part of the target or as part of the background. Studies are underway on how to include these effects properly in the modular framework of EOSTAR.
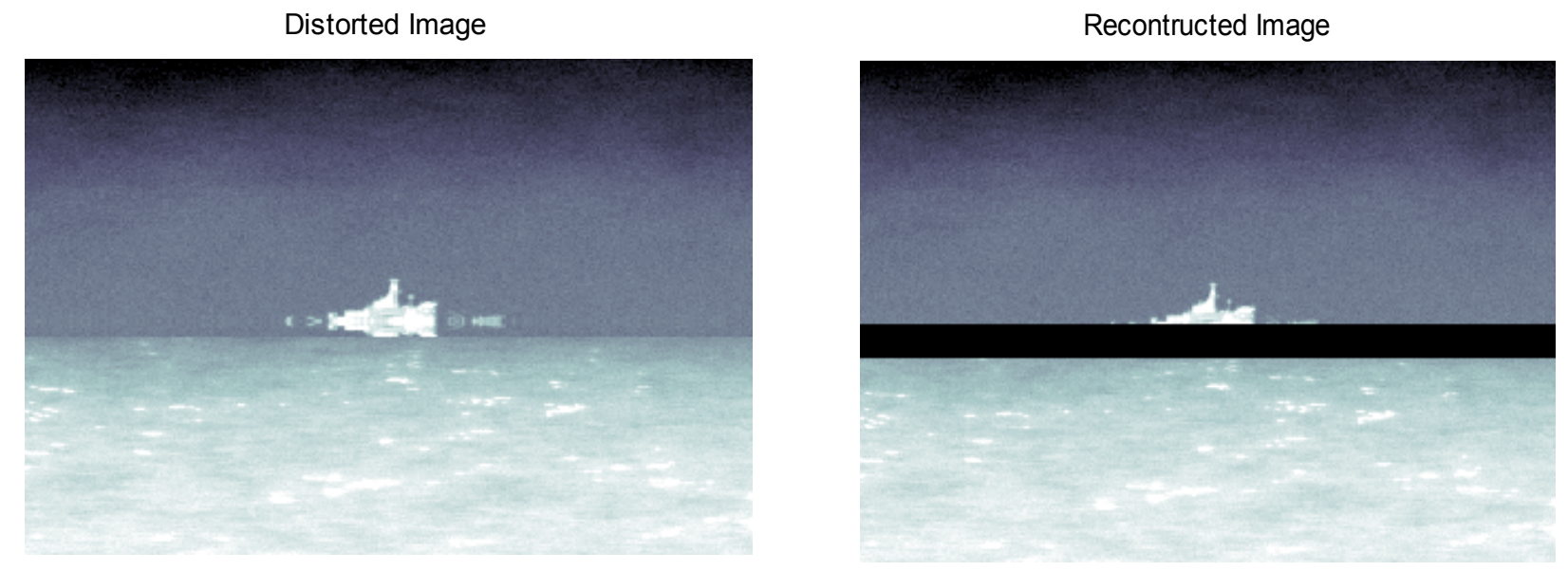

Figure 5: Left: synthetic image distorted by refraction effects. Right: reconstructed image. The image was reconstructed without a priori knowledge of the atmosphere. The black bar indicates the part of the target and background that could not be reconstructed. 


\subsection{Inverse methods / Image reconstruction}

As shown in Figure 1, EOSTAR includes a module "inverse methods". Inverse methods couple the result ("image on sensor") back to the forcing element, the environment. This allows retrieving information about the atmosphere from the actual imagery of the sensors deployed. Thus, inverse methods are not part of the forward end-to-end approach (main process in EOSTAR block diagram, see Figure 1), but a method to generate additional atmospheric input for the (realtime) end-to-end approach.

As an example, the left panel of Figure 5 shows a synthetic image of a ship with severe distortions due to refraction effects. This image was obtained via the forward route. Although inverse methods should be deployed on real sensor imagery, the synthetic image is useful for testing of the method as it generates a controlled environment.

Inverse methods rely on the unique relation between environmental conditions and sensor image. In the example of Figure 5, the distortions arise from specific gradients in the refractive index of the atmosphere, which in turn is a function of the vertical profiles of pressure, temperature and humidity. In the forward process, the impact of a particular refractive index profile on the image distortion is assessed with a ray-tracer (see section 2.3). In the inverse process, a mathematical technique ${ }^{10}$ is used to eliminate the distortions from the image and to retrieve the undistorted image and the profile of refractive index that caused the distortion. In this manner, the right panel of Figure 5 has been generated. As explained in [10], the target cannot always be fully reconstructed: the black bar indicates the part of the target and background that could not be reconstructed. Nevertheless, the reconstruction is appealing from an operational point of view, since it facilitates the classification / identification of the target by removing mirrored and deformed sections of the target.

As already mentioned, the inverse method also retrieves the refractive index profile. Since this profile at electro-optical wavelengths is largely determined by the temperature gradients, the inverse methods thus retrieve information about the temperatures in the atmosphere. In this way, inverse methods may complement / validate the atmospheric input data for the forward process. The advantage of the inverse methods is that the actual atmospheric state is probed through the sensor image.

\section{APPLICATIONS}

The modular approach in the EOSTAR model suite, as discussed in chapter 2, allows for a large degree of customization of the product for specific applications. This implies that EOSTAR can be (and currently is) utilized for quite a number of applications.

An important application for EOSTAR is the surveillance scenario, which necessitates insight in the effectiveness of the own sensors. The GUI shown in Figure 3 pertains to this use case. In surveillance mode, EOSTAR generates detection (classification, identification) ranges on the basis of the actual meteorological scenario. Alternatively, EOSTAR can provide a detection range forecast on the basis of a meteorological forecast, which is useful for mission planning.

When information about threat sensors is available, EOSTAR can also be used for platform survivability studies. In stand-alone applications, EOSTAR can be used to infer counter-detection ranges, e.g., of a periscope. In this manner, a signature management tool can be built, which allows evaluating the effect of changes in platform design or configuration on the counter-detection range. The TargetBuilder (section 3.1) is particularly useful in this application.

A further application of EOSTAR is training and education. The synthetic image allows operators to prepare themselves for the "live" images as displayed on their sensor during the mission. Furthermore, the operators can easily see the effect of a change in the environment (haze, wind), sensor setting (band selection), or target characteristics (speed, size) on the sensor's performance.

The kernel and underlying models can be provided as a DLL, which opens a range of applications for "EOSTAR inside". Here EOSTAR becomes a module for a larger model. Currently, EOSTAR is embedded in three larger applications. The first is a sensor fusion model providing recommendations for best sensor and sensor band selection, where EOSTAR provides the EO-sensor performance. The second is a war-gaming tool designed to evaluate mission accomplishment statistics for operational scenarios (again EOSTAR provides the EO-sensor performance). The third is an EO missile imaging seeker simulator to calculate target acquisition ranges and the effect of countermeasures. Here, EOSTAR provides the synthetic sensor image for the seeker simulator. ${ }^{11}$ 
The above paragraphs demonstrate the wide range of applications for which EOSTAR can be of value. The development of EOSTAR is not finished, since most of the above applications will need to mature. The development includes underlying scientific models, as well as user applications and (semi-)operational use of the model.

\section{ACKNOWLEDGEMENTS}

Major funding for the EOSTAR model suite, the underlying scientific models and the experimental data used in model development and validation was provided by the Royal Netherlands Navy, the Hydrographical and Oceanographic Service of the French Navy and the USA Office of Naval Research, Code 322 Marine Meteorology (Dr. Ron Ferek). The scientific EOSTAR project is a co-operation between TNO and Space and Naval Warfare Systems Center Pacific. The authors acknowledge their numerous colleagues at TNO who work and worked in code and model development, and the acquisition of experimental data underlying the models.

\section{REFERENCES}

[1] Schwering, P.B.W, "IRST evaluation methodologies: Maritime infrared background simulator". SPIE Proc. 620671, 620621 (2006).

[2] Neele, F.P., "Infrared ship signature prediction, model validation and sky radiance". SPIE Proc. 5811, 180-187 (2005).

[3] Http://www.ontar.com

[4] Van Eijk, A.M.J., Cohen, L.H., Navarro, L.J. and DeLeeuw, G., "Near-surface aerosol transmission in the marine environment". SPIE Proc. 4884, 160-169 (2002).

[5] Kunz, G.J., Moerman, M.M. and Van Eijk, A.M.J., “ARTEAM : Advanced Ray Tracing with Earth Atmospheric Models". SPIE Proc. 4718, 397-404 (2002).

[6] Kunz, G.J., "A bulk model to predict optical turbulence in the marine surface layer”, Report FEL-96-A053, TNO Physics and Electronics Laboratory, The Hague, The Netherlands (1996).

[7] Bijl, P., Hogervorst, M.A. and Vos, W., "Modular target acquisition model \& visualisation tool”. SPIE Proc. 6941, 69410C (2008).

[8] Penelon, T., Calmet, I., and Mironov, D.V., "Mircometeorological simulations over complex terrain with SUBMESO: a model study using a novel preprocessor". Int. J. Env. Poll. 16, 583-602 (2001).

[9] Pleber, T., "3D Targets in EO sensor performance evaluation". Report TNO-DV 2007 S397, TNO DSS The Hague, The Netherlands (2007).

[10] Degache, M.A.C, Van Eijk, A.M.J., Hammel, S.M. and Tsintikidis, D.S., "Analysis and deconstruction of mirages to assess environmental features". SPIE Proc. 7463, 74630D (2009).

[11] Schleijpen, H.M.A., "Toolset for evaluating infrared countermeasures and signature reduction for ships", SPIE Proc. 7814 (2010). 